\title{
Numerical Modeling of Disordered Foam in 3D: Effective Properties by Homogenization
}

\author{
C. Lusso ${ }^{1}$ and X. Chateau ${ }^{1}$ \\ ${ }^{1}$ Laboratoire Navier, UMR 8205, École des Ponts, IFSTTAR, CNRS, UPE, Champs- \\ sur-Marne, France.
}

\begin{abstract}
We investigate the numerical modeling and simulation of mechanical effective properties of disordered foams in $3 \mathrm{~d}$. We consider the elasticity, the thermal conductivity as well as the permeability. We present a meshing procedure for the microstructure of randomly disordered wet foams. The material behavior is modeled by constitutive equations and a boundary value problem is set on the microstructure. We present and implement the homogenization approach for the determination of the effective mechanical properties. The local problems are discretized and solved with the finite element method. We investigate the monodisperse and disordered configuration of a wet foam by varying the volume fraction.
\end{abstract}

\section{INTRODUCTION}

The structure of foam materials involves several length scales from macroscopic scale to macroscopic scale. In the context, the modeling approach is an homogenization, or multi-scale approach [Allaire, 1989], consisting in three main stages: the representation, the localization and the homogenization. In the first step, the modeling of the microstructure is established through a representative section of the material microstructure, namely a Representative Volume Element (RVE). The formal definition of the RVE requires a condition of separation of scales (micro-macro) assumed all along this work. The wet foam structure is modeled as a continuous network of convex polyhedral surfaces and the meshing procedure is based on a Voronoi tessellation, which in turn is based on random packings algorithm [Torquato et al., 2001]. The Voronoi tessellation then requires to evolve toward a physical structure of foam [Kraynik et al., 2003, Kraynik and Reinelt, 1999], this process is called the relaxation. In the localization stage, at the microscopic scale, the local properties on the RVE are derived as the solutions of a boundary value problem set on the RVE. We consider the deformation of the solid Plateau border, modeled by an elastic rheology. We also determine the thermal conductivity, the heat transfer is modeled by the Fourier's law on the Plateau border. Morevover, we study the fluid flow within the porous part. To model the microscopic flow within the pores, we consider the Stokes equations for a viscous 
fluid. These boundary value problems are discretized and solved with the Finite Element Method. Finally, the macroscopic mechanical properties are predicted by mean of averaging the local fields within the RVE.

\section{NUMERICAL MODELING OF THE FOAM STRUCTURE}

The structure of a dry foam is modeled as a set of convex polyhedra packed to fill space. We adopt the Voronoi tessellation algorithm to generate a partition of a sphere into convex polyhedral cells. Then, the surface of the Voronoi tessellation is drived to evolve toward physically relevant geometry modeling the foam microstructure [Kraynik et al., 2003, Kraynik and Reinelt, 1999]. This stage is called the relaxation [Kraynik et al., 2003, Kraynik and Reinelt, 1999] and the algorithm provides a stable structure of foam. The surface is evolved toward some local minimum in surface energy by iterating Conjugate Gradient descent method of optimization, with the Surface Evolver software developed by Ken Brakke (1992) [Brakke, 1992, Phelan et al., 1995]. Besides, the cells are constrained to have equal volumes, thus modeling a monodisperse structure of disordered foam. Furthermore, the mesh is regularized, the facets of the triangulation are shaped as equilateral as possible and each vertex is moved to the average position of its neighboring vertices. We iterate this algorithm until the number of nonminimal features (in Plateau's law [Weaire and Hutzler, 2001]) tends to 0. A wet foam structure can then be inferred starting from this relaxed structure. The extension to a wet foam structure with arbitrary volume fraction is achieved through the addition of the Plateau border. Subsequently the Plateau border's volume is gradually increased and adjusted. The volume meshing procedure is achieved with Gmsh [GMSH]. In the framework of the homogenization approach, the generated microstructure models the Representative Volume Element (RVE).

The figure 1 below depicted the mesh of a disordered microstructure with $N=$ 128 cells and a volume fraction $\phi=10 \%$. We represent both the Plateau border (left) and the porous part (right).

\section{STATEMENT OF THE LOCAL PROBLEMS}

\section{The elasticity problem}

The elasticity model is adopted to describe the small deformation of the solid Plateau border. The domain $\Omega_{s} \subset \mathbb{R}^{3}$ standing for the Plateau border is filled by a solid material. The rheology is defined by the linear Hooke's law and the constitutive equation reads:

$$
\sigma=2 \mu \varepsilon(\boldsymbol{u})+\lambda \operatorname{trace} \varepsilon(\boldsymbol{u}) \quad \text { in } \Omega_{s},
$$

where $\sigma$ denotes the total stress tensor, $\varepsilon$ the linearized strain tensor, $\lambda$ and $\mu$ stand for the Lam coefficients. We consider the equilibrium state of the material, the momentum 

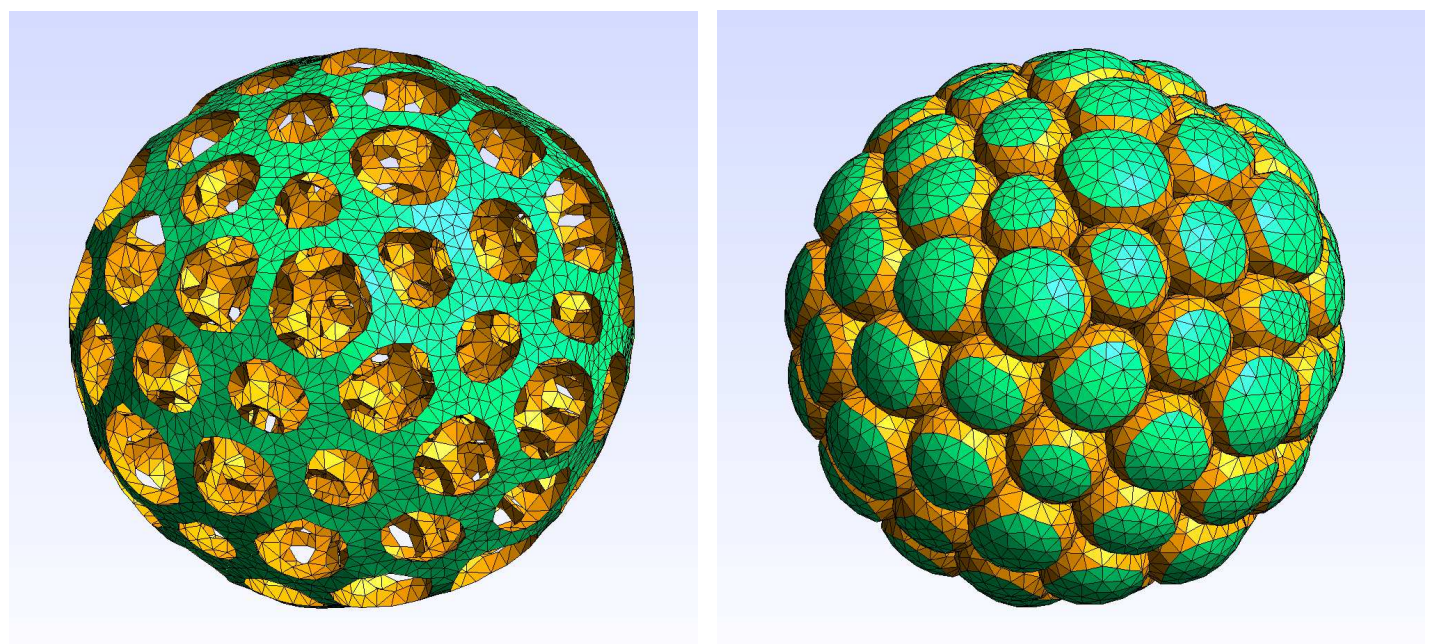

Figure 1. Meshed microstructure of a disordered foam with $N=128$ cells and a volume fraction $\phi=10 \%$. Plateau border (left) and porous part (right).

balance law with no body force reads

$$
\operatorname{div} \sigma=0 \quad \text { in } \Omega_{s}
$$

The boundary $\Gamma$ is divided into disjoint parts $\Gamma=\Gamma_{u} \cup \Gamma_{\sigma}$ denoting the external surface and the internal surface respectively, corresponding to the boundary where the displacement $\boldsymbol{u}$ is imposed and to the boundary where the traction $\sigma \boldsymbol{N}$ is imposed (nostress). In order to implement the homogenization approach, we apply an homogeneous loading $\boldsymbol{E}$ corresponding to uniform boundary conditions [Dormieux et al., 2002]. To achieve this we enforce

$$
\begin{aligned}
\boldsymbol{u} & =\boldsymbol{E} \cdot \boldsymbol{x} & & \text { on } \Gamma_{\boldsymbol{u}}, \\
\sigma \boldsymbol{N} & =0 & & \text { on } \Gamma_{\sigma},
\end{aligned}
$$

where $\boldsymbol{N}$ stands for the outward unit normal on $\Gamma$ and $\boldsymbol{E}$ is an order 2 tensor.

\section{The thermal conductivity problem}

The heat transfer model on the solid Plateau border is employed in order to prescribe the thermal conductivity. The behavior is defined by the linear Fourier's law and the constitutive equation reads

$$
\boldsymbol{q}(\boldsymbol{x})=-\kappa \nabla T(\boldsymbol{x}) \quad \text { in } \Omega_{s},
$$

where $T(\boldsymbol{x}) \in \mathbb{R}$ denotes the temperature within the material, $\boldsymbol{q}(\boldsymbol{x}) \in \mathbb{R}^{3}$ is the heat flux and $\kappa$ is the conductivity coefficient. We consider the thermal equilibrium state of 
the material, the thermal balance law is expressed as

$$
\operatorname{div} \boldsymbol{q}(\boldsymbol{x})=0 \quad \text { in } \Omega_{s} .
$$

The boundary $\Gamma$ is divided into disjoint parts $\Gamma=\Gamma_{T} \cup \Gamma_{\boldsymbol{q}}$. In order to implement the homogenization approach, we apply an homogeneous loading $\boldsymbol{A}$ corresponding to uniform boundary conditions. To achieve this, we enforce

$$
\begin{aligned}
T & =\boldsymbol{A} \cdot \boldsymbol{x} & & \text { on } \Gamma_{T}, \\
\boldsymbol{q} \cdot \boldsymbol{N} & =0 & & \text { on } \Gamma_{\boldsymbol{q}},
\end{aligned}
$$

where $\boldsymbol{A}$ is an order 1 tensor. The solution represents the local flux $\boldsymbol{q}$ and the local temperature $T$ at the microscopic scale, induced by the macroscopic loading $\boldsymbol{A}$.

\section{The Stokes problem}

At the microscopic scale, the flow of a viscous fluid is governed by the Stokes equations [Allaire, 1989]. The domain $\Omega_{f} \subset \mathbb{R}^{3}$ standing for the porous part saturated by an incompressible Newtonian fluid. The constitutive equation is written

$$
\sigma=2 \mu D(\boldsymbol{u})-p \quad \text { in } \Omega_{f},
$$

where $\boldsymbol{u} \in \mathbb{R}^{3}$ denotes the velocity, $p \in \mathbb{R}$ is the pressure field and $\mu>0$ is the viscosity. We consider the equilibrium state of the material and the fluid is assumed to be incompressible.

$$
\begin{aligned}
-\mu \operatorname{div} D(\boldsymbol{u})+\nabla p=0 & & \text { in } \Omega_{f}, \\
\operatorname{div} \boldsymbol{u}=0 & & \text { in } \Omega_{f} .
\end{aligned}
$$

The boundary of the domain is disjoint into $\Gamma=\Gamma_{\mathrm{sf}} \cup \Gamma_{e}$, where $\Gamma_{\mathrm{sf}}$ stands for the solidfluid interface and $\Gamma_{e}$ is the external surface. We assume a no-slip boundary condition at the solid-fluid interface and the homogenization approach is implemented by applying an homogeneous loading $\alpha$ on the RVE.

$$
\begin{aligned}
\boldsymbol{u} & =0 & & \text { on } \Gamma_{\mathrm{sf}}, \\
\sigma \boldsymbol{N} & =-(\alpha \cdot \boldsymbol{x}) \cdot \boldsymbol{N} & & \text { on } \Gamma_{e} .
\end{aligned}
$$

\section{NUMERICAL RESULTS}

The problems, and are written in a variational form and discretized using the Galerkin method together with the finite element method. The resolution is achieved throught the FreeFem++ langage and the numerical method is wether the conjugate gradient or the GMRES solver for larger systems such as the $N=512$ cells mesh. 

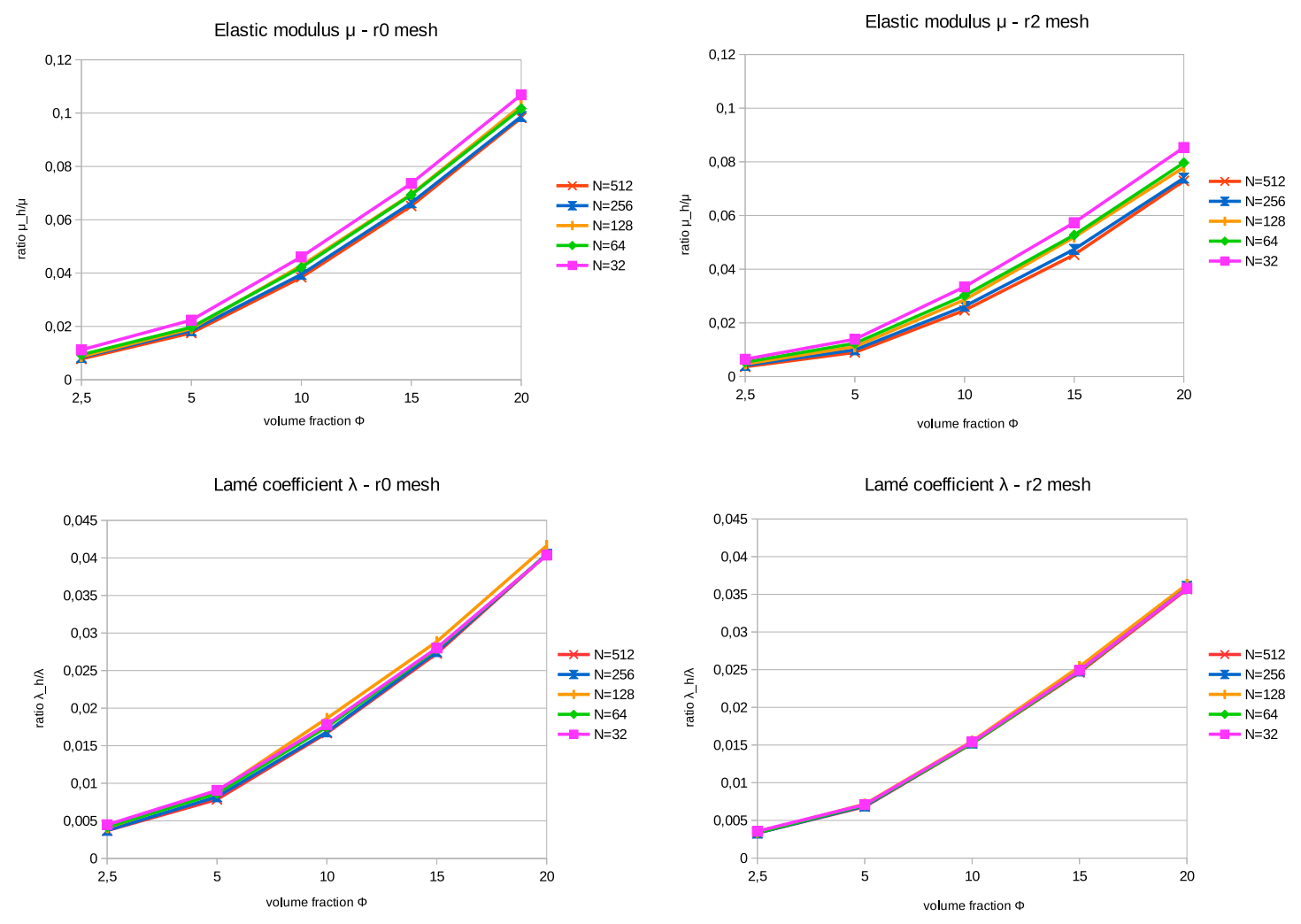

Figure 2. Elastic modulus $\mu_{h}$ normalized with $\mu=\frac{E}{2(1+\nu)}=\frac{1000}{2,6}$ and $\operatorname{Lam} \tilde{\mathbf{A}} \odot$ coefficent normalized with $K=\lambda+\frac{2}{3}=\frac{1000}{1,2}$, with respect to the volume fraction $\phi$. Left: no refinement $\left(r_{0}\right)$ and right: 2 refinements $\left(r_{2}\right)$

\section{Elasticity}

We have determined the macroscopic properties by averaging the stress field on the whole domain. As the structure exhibits isotropy, the discrete macroscopic elastic tensor $\mathbb{C}_{h}$ is reduced to 3 coefficients $\lambda_{h}+2 \mu_{h}, \lambda_{h}$ and $\mu_{h}$ where $\lambda_{h}$ and $\mu_{h}$ are the discretized Lam coefficients. In the figure 2 below, we represent $\mu_{h}$ and $\lambda_{h}$ with respect to the volume fraction $\phi$. We have compared the result for different number of cells $N$, on both a $r_{0}$ (no refinement) mesh and a $r_{2}$ (2 refinements) mesh.

The macroscopic properties linearly increase with the volume fraction. The size of the RVE affects the results mainly for the elastic modulus. For the Lam coefficient, on the $r_{2}$ mesh, the converged homogenized solution is reached even with $N=32$ cells, whereas for the elastic modulus, the converged homogenized solution is not reached even with $N=512$ cells. In other words, with two refinements, the size of the RVE is large enough to obtain the converged homogenized solution $\lambda_{h}$. In addition, by studying the periodic case of a Kelvin structure, we have inferred that our result with $N=512$ are about $20 \%$ different from the converged homogenized solution. 

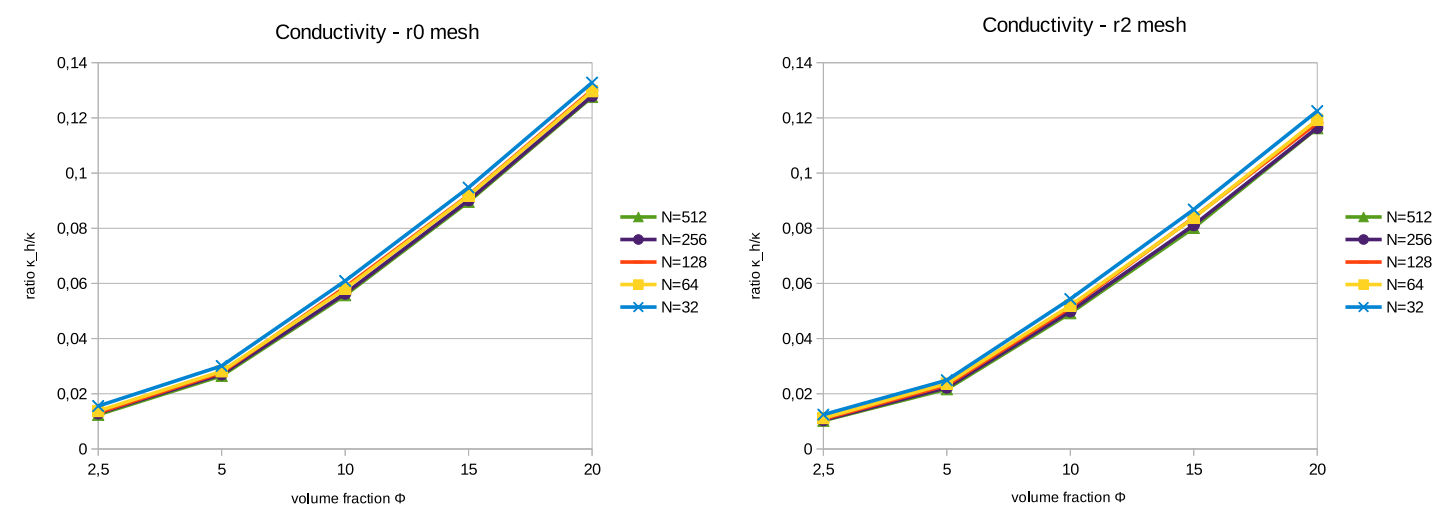

Figure 3. Thermal conductivity $\kappa_{h}$ normalized with the conductivity coefficient $\kappa=100$. Left: no refinement $\left(r_{0}\right)$ and right: 2 refinements $\left(r_{2}\right)$.

\section{Thermal conductivity}

We have determined the macroscopic thermal conductivity by averaging the flux field on the whole domain. As the structure exhibits isotropy, the discrete macroscopic thermal conductivity tensor $\mathbb{K}_{h}$ is reduced to $\kappa_{h}$, where $\kappa_{h}$ is the discretized conductivity. In the figure 3 below, we represent $\kappa_{h}$ with respect to the volume fraction on both the $r_{0}$ mesh and the $r_{2}$ mesh.

The macroscopic thermal conductivity linearly increases with the volume fraction. The size of the RVE slightly influences the results since we obtain almost superimposed results with respect to the number of cell $N$, and the results are enhanced with two refinements (our approximation provides an upper bound of the homogenized solution). Meaning that the numerical error of approximation dominates the error of the homogenization approach. In addition, by studying the periodic case of a Kelvin structure, we have inferred that our result with $N=512$ are about $5 \%$ different from the converged homogenized solution.

\section{Permeability}

The resolution is achieved through a MPI (Message Passing Interface) formulation in FreeFem++ langage with a MPI resolution on $n_{p}=4$ processors. The numerical method adopted to solve the linear system is the Mumps (MUltifrontal Massively Parallel Solver) which is a sparse direct solver. We determine the macroscopic permeability by averaging the velocity field on the whole domain. As the structure exhibits isotropy, the discrete macroscopic permeability tensor $\mathbb{K}_{h}$ is reduced to $\mathbb{K}_{h}=k_{h}$, where $k_{h}$ is the discretized permeability. In the monodisperse configuration, the average cell volume $v_{B}$ stands for the only characteristic length scale. In the figure 4 below, 


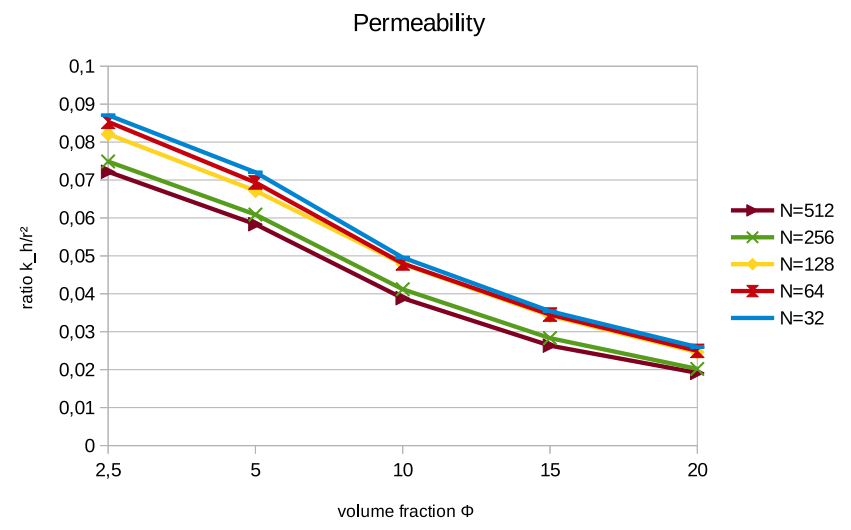

Figure 4. Permeability $\frac{k_{h}}{r^{2}}$ with respect to the volume fraction $\phi$.

we represent the ratio $\frac{k_{h}}{r^{2}}$, where $r=\left(\frac{3 v_{B}}{4 \pi}\right)^{\frac{1}{3}}$, with respect to the volume fraction. We compare the result for different value of number of cell $N$.

The macroscopic permeability linearly decreases with the volume fraction. The size of the RVE significantly influences the results. More specifically, we observe that the number of cell has more influence on low volume fraction $\phi=2,5 \%$ than on high volume fraction $\phi=20 \%$. This fact relies on the slightly higher number of elements for meshes with $\phi=20 \%$, leading to a more accurate approximation of the numerical solution in the finite element method.

\section{CONCLUSION}

First of all, we point out that an elaborate meshing procedure has to be carried out, which is time consuming and difficult to implement in the case of real microstructures. Indeed, the microstructure of foams presents irregularities such as singularities and requires to reach a stable state of equilibrium, modeling the physically relevant microstructure of foams. In this context, we manage to entirely automate the foam meshing procedure of disordered wet foams. Besides, our results are in good agreement with the well-known results on dependence of the mechanical properties of the foam with respect to the volume fraction (we have compared with estimates available in the literature data). As a consequence, we are confidant that a 512 bubbles RVE with two refinements mesh provides an accurate homogenized solution.

\section{ACKNOWLEDGMENTS}

We gratefully acknowledge financial support from Agence Nationale de la Recherche (Grant no. ANR-13-RMNP-0003-01). 


\section{REFERENCES}

[Allaire, 1989] G. Allaire, homogenization of the Stokes flow in a connected porous medium, Asymptotic Anal. 2, 3 (1989), 203-222.

[Brakke, 1992] K. Brakke, The Surface Evolver, Experimental Math. 1 (1992), 141165.

[Phelan et al., 1995] R. Phelan, D. Weaire and K. Brakke, Computation of equilibrium foam structures using the Surface Evolver, Experimental Math. 4 (1995), 181192.

[Dormieux et al., 2002] L. Dormieux, A. Molinari and D. Kondo, Micromechanical approach to the behavior of poroelastic materials, J. Mech. and Phys., Elsevier, 50 (2002) 2203-2231.

[GMSH] C. Geuzaine and J.-F. Remacle, Gmsh: a three-dimensional finite element mesh generator with built-in pre-and post-processing facilities International Journal for Numerical Methods in Engineering, 79, (2009), 1309-1331.

[Hecht, 2012] F. Hecht, New development in FreeFem++, J. Numer. Math. 20 (2012), 251-265. http://www.freefem.org/ff++

[Kraynik et al., 2003] A.M. Kraynik, D.A. Reinelt and F. van Swol, Structure of random monodisperse foam, Phys. Rev. E, 67 (2003).

[Kraynik and Reinelt, 1999] A.M. Kraynik and D.A. Reinelt, Foam microrheology: from honeycombs to random foams, Technical Report, Engineering Sciences Center MS 0834, Sandia National Laboratories and Department of Mathematics, Southern Methodist University (1999).

[Torquato et al., 2001] S. Torquato, Random heterogeneous materials: microstructure and macroscopic properties, pringer-Verlag, New York (2001).

[Weaire and Hutzler, 2001] D.L. Weaire and S. Hutzler, The Physics of Foams, Clarendon Press (2001). 